\title{
Emotional Reactions to Sports Heroes' Rise and Fall: Application of Affective Disposition Theory Via the Hero Narrative
}

\author{
Sue Ellen Christian ${ }^{* 1}$ and Leah M Omilion-Hodges ${ }^{2}$
}

Associate Professor, Journalism, University of Michigan, USA

School of Communication, Western Michigan University, USA

"Corresponding author: Sue Ellen Christian, Associate Professor, Journalism, University of Michigan, USA, Tel: (269) 387-3145; E-mail: sueellen.christian@wmich.edu

Rec date: May 08, 2014; Acc date: June 10, 2014; Pub date: June 17, 2014

Copyright: (c) 2014 Christian SE, et al. This is an open-access article distributed under the terms of the Creative Commons Attribution License, which permits unrestricted use, distribution, and reproduction in any medium, provided the original author and source are credited.

\begin{abstract}
Researchers have contended that the American news media often depict celebrity athletes as the heroes in a narrative that has a schematic familiarity for consumers. The presentation of the athlete at the center of the hero narrative follows a sequence of events operationalized by Hoebeke, Deprez, and Raeymaeckers in 2011. This study assesses the extent that the audience perceived, accepted and retold the hero narrative of cyclist Lance Armstrong and collegiate football player Manti Te'o in the winter of 2012, when both athletes were facing public crises. This study measured the dynamics of Affective Disposition Theory by assessing participants' levels of empathy/ sympathy, liking and identification with the athletes. We discuss the findings in the context of news consumption of the hero narrative as predictive of ADT.
\end{abstract}

Keywords: Affective disposition theory; Celebrity athletes; Hero narrative; Schema; News consumption

\section{Introduction}

Daily news offers myth to the masses, serving up archetypal stories on a regular basis [1], and among the most popular subjects for such stories are athletes [2,3]. One way that news outlets create this mythology around athletes is through the hero narrative [4]. Transforming athletes into the archetypal hero involves a sequence of events-humble beginnings, athletic achievements, obstacles faced and overcome, a short-term fall or failure, and a return to good graces-that the news media details in narrative fashion [4]. Our research explores how the audience assimilates the hero narrative and internalizes it as their own. We do this by uniting the hero narrative with affective disposition theory (ADT); ADT presumes that the news media create certain schemas for news consumers, and in doing so, establish consumer expectations about how an athlete's narrative should unfold [5].

One reason that news audiences are entertained or engaged by a particular news story is because they feel an emotional connectionwhether positive or negative-to the subject of the story. ADT is undergirded by the concept of connection. ADT holds that observers' affect toward media characters influences their appreciation of content about those characters [6]. This theory has been successfully applied to content involving news, theatre, humor and sports. For example, Zillmann, Taylor, and Lewis [7] found that stories with individuals as opposed to topics (such as foreign affairs) elicited expected results. That is: the more a news consumer liked a news figure, the less amusing and enjoyable were the revelations about his/her victimization. The reverse was also true: the more a news figure was disliked, the more amusing and enjoyable were such revelations. The researchers [7] suggested "Affective dispositions convert normative goodness and badness into subjectively good or bad news such that good and bad news, then, is 'in the eyes of the beholder,' rather than inherent in the events of a report". Zillmann, Bryant and Sapolsky reiterate that their findings of sports fans' connection to their players and teams "leave no doubt that people 'have feelings about athletes,' and that they enjoy or despise events and outcomes in accord with their feelings".

Specifically, this inquiry seeks to understand the communication of sports figures in light of the hero narrative while utilizing ADT to understand how members of the college-age audience form value judgments about fallen athletes, which in turn leads to emotional responses [8]. This project seeks to broaden the field's understanding of how audiences form personal attachments with and attach values to sports characters. This understanding is particularly relevant at a time when sports interest, sports consumption and sports' role in a capitalist economy are as vital as ever $[9,10]$. Moreover, members of the college-age audience are among the most avid global consumers of sports and sports news; $29 \%$ of fans that follow sports via an Internetabled mobile device are 18 to 24 years old, and $37 \%$ of sports fans in the U.S. who followed sports via a social network platform are between 18 and 24 [10]. Historically in time Lance Armstrong and Manti Te'o were centers of attention conveniently providing a pair of archetype athlete cases to study to nuance the field's knowledge of ADT as influenced by the hero narrative in a college-age audience. We found the coincidence of their public crises in the news at the same time an opportunity for inquiry into how those differences might play out via an exploration of ADT. Examination of the Armstrong and Te'o cases also yields insight into how thoroughly news consumers ingested powerful but typical narratives about news figures-in this case-the athlete-as-hero narrative.

Armstrong and Te'o were selected for study because they both achieved significant athletic accomplishments and were also the focal point of media attention and fan judgment and a broad range of fan reactions [11,12]; for drug use in the case of Armstrong and a fictitious online girlfriend in the case of Te'o. Despite clear commonalities, the 
athletes also differ in important ways. Armstrong is a longtime athlete, older and in an individual sport. Te'o is a rising star, young and in a team sport. Anecdotally, these two athletes are compelling characters for the college-age audience: The "Catfish" phenomenon that the Te'o case depicts may well have caught the attention of the college-age audience, as even the sophomore season of the MTV docu-series drew 2.5 million viewers, securing the highest ratings in the 12 to 34 age demographic [13].

Examination of ADT and the hero narrative will a) contribute to the utility of the hero narrative paradigm, b) add to the body of ADT knowledge, and c) nuance the field's understanding of the narrative messages that college-age consumers absorb. We begin with a thorough examination of the hero narrative as schema in ADT before exploring the emotional reactions and connections consumers form with those in the public eye. The discussion addresses theoretical implications in addition to briefly raising relevant pragmatics such as the role of journalists in furthering the hero narrative and the communication of athletes' persona and sports marketing.

\section{The Hero Narrative as Schema in ADT}

ADT presumes the development of attitudes about characters (be they in the news, athletic arena or theater), such that audience members anticipate characters' outcomes based on their judgments about the characters $[6,14,15]$. The theory suggests that the news media create schemas for news consumers, helping viewers to form expectations about how a particular athlete's or news figure's narrative should unfold [5] whereas how an athlete's narrative should unfold is explicated in the hero narrative. Athletes are popular subjects for archetypal stories in the mass media $[2,3]$ due in part to the mythology in which news outlets steep sports figures. One way that news outlets create these legends is through the hero narrative [4]. As mentioned, the hero narrative template involves a notable and successful athlete incurring a fall or failure before ultimately returning to public acceptance [4].

The hero narrative is a powerful mechanism [16]. The repetition of the hero narrative in news accounts creates a schema for news consumers. A schema represents a cognitive framework or concept that helps organize and interpret information. It allows people to intake new information and "match" it with existing information in their brain that is related or familiar. The more that people consume about a celebrity athlete, the more they ingest the story schema that includes expectations about how a narrative unfolds [5]. As Raney [14] put it, repeated media exposures teach viewers how stories are constructed and themes are repeated, such that "viewers arguably do not encounter most narratives empty handed, or empty minded".

The hero narrative is in many ways like a Disney fairy tale; the characters may be different, but the plot structure is the same $[8,17]$. In this way, a schema, or cognitive framework, about the story line develops and audience members can insert the character-or in the present study, the athlete-into the familiar narrative arc. The news media are adept at following a narrative structure that is at once familiar and that calls upon schemas as to what should happen next and to whom in the narrative consumers should affiliate with or cheer for, even when characters are on the wrong side of good. Interestingly, in Raney's [8] application of ADT, he argued that organized prior knowledge about a category or a schema, helped viewers decide whether they liked a character or not. The repetition of familiar narratives such as the hero myth as applied to sports celebrities means that schemas are activated and these schemas guide expectations and interpretation of the characters involved in an ongoing story [14].

\section{The hero narrative and consumer emotions}

Just as familiar narratives, such as Disney stories are meant to engage viewers' emotions with heroes and villains, so too the application of ADT via the hero narrative shows how the narrative elicits judgment and emotional reactions. For example, Lewis and Proffitt found in their analysis of three athletes' use of marijuana, Michael Phelps was often portrayed as a hero and role model even despite his use of illegal substances. Similarly, news organizations have emphasized the narrative/story element in articles such that accounts are both informative and entertaining [18] and the mediated construction of an athlete as a hero has existed for some time [19]. Hoebeke and colleagues [4] showed that journalists are adept at telling the hero narrative in all its ups and downs suggesting, "Both myth and news stories can be considered moral tales. Hoebeke et al. [4] forwarded nine narrative sequences of the hero journey: Rise, which includes humble descent, quest, obstacles and ordeals, important victories; Temporary fall from grace: trickster, which includes savage or victim; and Resurrection, which includes the chance to defend or explain mistakes, penance accepted, and a return as media turns positive again. In addition, the archetype includes the status elements of celebrity or role model, and the social values the hero must represent. The researchers [4] were able to successfully apply the hero narrative to Belgian cyclist Tom Boonen, who came under scrutiny when it was learned that he abused drugs. Prior, Boonen was depicted as a role model, treated as a celebrity with the attendant focus on his personal life and his bankability. However, because his drug use was part of his private life, this allowed the news media to portray Boonen as a hero who abided by the rules of his sport. To a limited extent, the authors captured the news consumers' reactions through quotes in news stories, noting that fans had forgiven Boonen and were supportive of second chances-and in so doing, the natural connection between the hero narrative and ADT was illustrated.

\section{Affective Disposition Theory}

The success of the hero myth can be explained by ADT [20]. ADT creates expectations about characters or, for present purposes, athletes. Consumers in turn form expectations of outcomes for these characters based on evaluations of athletes' behaviors and personalities. Even when characters are moral ambiguous, consumers enjoy them [21]. Audiences' favorable dispositions toward public figures heighten enjoyment when good things happen to those figures, and dampen reactions when bad things happen to them [7]. Schafer and Raney [20] xplored how audiences can still enjoy antihero figures, again utilizing story schema to facilitate this affective experience. Viewers are constantly evaluating characters' actions and their outcomes and the role the characters played in those outcomes in order to make judgments about the characters [22]. ADT explains the powerful connection that audiences form with those in the public eye, including prominent athletes.

For example, Kinnally, Tuzunkan, Raney, Fitzgerald, and Smith [23] applied both the schema-triggered effect model and ADT to depictions of athletes in media coverage. The schema-triggered effect model states that a person understands of another helps inform their feelings toward that person. Their study lent support to Raney's [6] postulation that at least initially, schemas can trigger dispositional affect more than an independent moral assessment of actor's behaviors 
and motivations. Schemas, then, serve to prime an observer's tendencies by awakening already-stored concepts, even feelings, and storylines, including the oft-used hero narrative as applied to sports figures.

Fortunately, the theoretical road is well paved in this regard. By example, Aust concluded that observers would respond empathetically when disposed to liking the subject of their observation. Others Kinnally et al. [23] have applied ADT to fictitious sports figures; according to the authors, sports news was akin to news content that informed audiences of the personality, behavior and motivations of athletes off the field which corroborates other applications of ADT $[24,25]$. Taken together, these recent studies reinforce the importance of exploring consumers' feelings of empathy toward the athlete-hero and help to explain why consumers may come to identify with sports figures.

\section{Identification}

Research continues to reiterate the social nature of human beings as genetically wired to crave attachments to others [26]. These attachments may be in the form of face-to-face or socially-mediated relationships, but may also occur in less intimate encounters such as between a media consumer and a television character or a popular figure such as an athlete [27]. For example, Kassing and Sanderson [28] found that fans of Floyd Landis saw the cycler as a drinking buddy. Relationships of this nature are referred to as parasocial interactions since the association feels like a "face-to-face relationship" to one of the actors [29]. While parasocial describes a one-sided relationship that occurs only in the mind of a media consumer, such attachments can result in powerful bonds between an audience member and a media target, such that audience members may even alter their behavior due in part to the perceived relationship [30]. Concerning this, participants who disclosed a parasocial relationship with either Magic Johnson or Princess Diana reported varied health behaviors compared to those who did not [31]. A large component of the parasocial relationship rests on consumers' perceptions of similarity with the media figure, including active comparisons resulting in a deeper-rooted source of identification of the consumer with the figure [32]. To that end, identification occurs as parasocial relationships mature.

Identification with individuals or characters presented in the media transcends simple spectatorship to detail a more intimate action. That is, instead of objectively consuming a sports figure's narrative, individuals who identify with the figure are likely to internalize the feelings and experiences of the athlete [33]. Those who identify with a figure in the media are thought to vicariously participate in the athlete's experiences [34] and "psychologically merge with the persona" [30]. Consumers who identify with media figures will often engage in active fantasies in which they imagine themselves embodying the identity of the target [28]. Considering this, parasocial relationships fuel consumer identification with media figures, in which identification allows viewers to feel and live the experiences and emotions of the figure. In this sense, consumers may witness coverage of a sports figure and enjoy their successes, mourn their losses, and blush with their indiscretions.

Turning to this study's main characters-Lance Armstrong and Manti Te'o-we seek to explore how particular indiscretions or a fall from grace, as the hero narrative would posit, affect the ways in which viewers identify with athletes. Armstrong, an internationally renowned athlete, philanthropist, and cancer survivor, repeatedly countered allegations of illegal drug use by lying to media and fans. Thus, in Armstrong's case, he actively constructed and maintained fallacies in order to protect not only his reputation, but to preserve his sports records. This is profoundly different from Te'o, whose downfall occurred via a common medium - the Internet. As noted, Te'o was often cast as a pawn lured into a cruel hoax via fabricated media exchanges. Considering that study participants (i.e., undergraduate students) often cite the Internet as their medium of choice and the first place they access sports news [10], we suggest:

\section{Armstrong.}

H1: Participants are more likely to identify with Te'o than with

Despite the turmoil associated with identifying with a sports figure, particularly one whom the media position within a hero narrative, consumer may remain committed to athletes simply because they like them and feel an emotional connection.

\section{Emotional reactions}

Research indicates that consumers, particularly fans, may incur a large range of emotional reactions while following athletes, including empathy. Empathy is both affective and cognitive in nature and involves mirroring another's mental state. Hogan concluded that it is akin to apprehending another person's state of mind even when not in reality experiencing the person's emotions. Empathy is also affective, engaging the consumer's emotions as he or she perceives them to be experienced by another [35]. The latter is more visceral and automatic; the first is more intellectual in nature.

Empathy is central to ADT, such that a variety of empathetic responses felt toward sports figures led to varied responses from news consumers [6]. Zillman and Paulus' review of research on dispositional reactions by spectators to athletic performance shows a rich emotional connection between the audience of a sporting event and the athletic performers. Lewis and Proffitt examined the various news writers' depictions of swimmer Michael Phelps as compared to three other high-profile athletes who also used marijuana. The authors note that it helps when an athlete is a winning athlete: Phelps' success in the pool seemed to buy him a pass on his marijuana use when it came to the press' depictions of athlete misconduct. Phelps was described as a hero, a contrast to the struggling cases of Michael Vick and Josh Howard. With regards to these findings, we are curious as to whether the emotion of empathy, which requires the sense of walking in another's shoes to share their trials, might be more apparent for Te'o than for Armstrong. At the same time, we acknowledge the years of consumption of the hero narrative with Armstrong as main character, and Armstrong's status as a cancer survivor and global spokesperson for cancer research funding. So we pose the following research question:

RQ1: How will participants differ in their empathy toward Armstrong and Te'o?

Relatedly, Shafer and Raney [20] explored the power of ADT by applying it to the anti-hero narratives that are increasingly populating television shows and films. As they note, consumers' moral reactions to characters, their successes or failures and their outcomes, determine how much consumers like, hope for, and enjoy those characters. Further, athletes, in comparison to other media figures, were particularly susceptible to parasocial relationship violations in which consumers, especially women, reported decreasing their level of intimacy of their association with the athlete [36]. Considering this, relationships with athletes is an area ripe for study because consumers 
identify, develop relationships, have belongingness needs met, and behave in relationally anticipated ways with these media figures. However, as previous studies indicate [30,34,35] questions still linger regarding how ADT influences consumer perceptions. To that end, the authors forward hypotheses that seek to answer these questions by examining consumers' emotional reactions to two notable athletes, Armstrong and Te'o.

Within the hero narrative Te'o didn't fall as far from grace as Armstrong. The fall he experienced was less of his doing; he was passive, at least at the start of the virtual relationship. Armstrong, by contrast, was active in his deceit, lying repeatedly to the public via the news media and advertising that he was clean of performanceenhancing drugs. Despite the hero narrative that both athletes arguably have been cast in by news coverage, it is likely that consumers will be more sympathetic to Te'o, whom many news articles depicted as the victim of a cruel hoax $[37,38]$ rather than as perpetrator. So, we assert:

H2: Participants will be more likely to feel sympathy toward Te'o than Armstrong.

In conjunction with the second hypothesis, we predict that because he was a victim, Te'o will be seen as more gullible and naive than Armstrong. In the same vein, it is likely that Armstrong will be perceived as more cunning and manipulative. Thus, the following hypotheses are posed:

H3a: Participants are likely to perceive Te'o as more naïve than Armstrong.

H3b: Participants are likely to perceive Te'o as more gullible than Armstrong.

H3c: Participants are likely to perceive Armstrong as more manipulative than Te'o. Te'o.

H3d: Participants are likely to perceive Te'o as more cunning than

Moreover, extant literature (e.g., Bush, Bush, Clark, \& Bush, Sargent, Zillmann, and Weaver) has indicated that in some instances, gender may play a decisive role in participants' identification and emotional reactions to a sport or celebrity/sports figure. Because of these findings, we forward a second research question:

RQ2: How, if at all, does gender influence participant identification, empathy and sympathy toward Armstrong and Te'o?

\section{Method}

This study employed a quasi-experimental research design in which participants were randomly selected into either the Armstrong or the Te'o condition. After being randomly assigned to a condition, participants had to self-identify as news consumer familiar with their respective athlete in order to complete the study. This ensured that all participants were aware of the scandal surrounding the sports figure on whom they were randomly assigned to comment. Participant demographics and study procedures are detailed below.

\section{Participants and procedures}

Some 166 undergraduate communication students from a large, Midwestern university participated in this empirical study. On average, participants were 21 years old $(S D=2.18)$; women $(n=99)$ composed the majority of the sample and men made up the remaining
$40 \%(n=67)$. Also, $9 \%$ of the sample were freshman $(n=15), 13 \%$ sophomores $(n=21), 52 \%$ juniors $(n=87)$, and $26 \%$ seniors $(n=43)$. In regard to ethnicity, the majority of participants $(61 \%, n=101)$ were Caucasian, followed by $20 \%(n=34)$ African American/Black, with the remaining $19 \%$ of participants Hispanic $(n=10)$, Asian $(n=2)$ or other $(\mathrm{n}=19)$ heritage.

After securing HSIRB approval, the researchers recruited participants in communication courses in which they were awarded a nominal amount of extra credit for taking part in the online, anonymous study. After reading the online information sheet about the study parameters and purpose, participants were randomly assigned to either the Armstrong or Te'o condition. Once participants were randomly placed into one of the two conditions, they were immediately asked to indicate whether or not they were familiar with the recent news events involving Lance Armstrong or Manti Te'o. Participants who acknowledged that they were familiar with the athlete in question were retained for this study. Participants who selfidentified as unfamiliar with either Armstrong or Te'o were redirected to an outside Associated Press story and then presented with the study's instruments. However, those individuals are not included in the current study $(\mathrm{n}=17)$, because overwhelmingly, participants acknowledged a familiarity with the athlete in question. In sum, participants retained for this study unanimously self-identified as a consumer who had knowledge of the sports figure in question. Approximately $58 \%(\mathrm{n}=96)$ of participants indicated their perceptions of Armstrong while $42 \%$ were randomly assigned to Te'o condition $(n=70)$.

\section{Instrumentation}

Unless otherwise noted, all scales utilized a seven-point Likert-type scale ranging from strongly disagree to strongly agree. Additionally, confirmatory factor analysis was conducted to verify the psychometric properties of each scale.

\section{Empathy and sympathy}

Four items originally developed by Baker were utilized to measure the amount of empathy and sympathy participants felt toward their respective sports figure. The empathy scale was created with the intention of understanding the positive and negative experiences of another. Sample items to assess empathy include, "Seeing Te'o in this situation made me feel distressed" and "When I saw Armstrong in this situation I felt protective toward him." This scale demonstrated high internal consistency $(\alpha=.86)$, which aligns with previous uses of the scale (i.e., Baker). The sympathy scale sought to examine the actual emotional responses participants experienced in part due to the perceived suffering of the sports figure. Sample items include, "I felt sorry for Te'o" and "Armstrong had my sympathy." The sympathy scale also indicated acceptable reliability with a Cronbach alpha of .83 .

\section{Social attraction}

McCroskey and McCain's 1974 social attraction subscale was utilized to measure how closely participants identified with their respective sports figure [39]. "I would like to have a friendly chat with Te'o" is a sample item from the social attraction scale. As it has previously, [40] the scale has again indicated high reliability $(\alpha=.84)$. 


\section{Personality traits}

The authors forwarded four additional items to measure perceptions of personality traits of the two athletes: gullible, manipulative, cunning, and naive. A sample question includes, "I believe that Te'o is gullible."

\section{Results}

Correlational and t-test analyses were conducted to explore the study's research question and hypotheses.

Hypothesis one suggested that participants would be more likely to identify with Te'o more than Armstrong. However, independent samples t-test indicated that the average social identification score for Te'o $(M=4.08, S D=1.22)$ was approximately the same as participants' identification with Armstrong $(\mathrm{M}=4.01, \mathrm{SD}=1.12)$, indicating that hypothesis one was not supported.

The first research question and second hypothesis revolved around participant empathetic and sympathetic responses. Considering the amount of emotional turmoil each athlete incurred, a research question was posed in regard to empathy, whereas hypothesis two proposed that participants would feel greater levels of sympathy toward Te'o than Armstrong. Interestingly, participants did not vary significantly in their perceptions of empathy for either athlete. While both athletes were above the scale mean for empathy $(M=3.50)$ and generally participants were more empathetic toward Te'o $(\mathrm{M}=4.29$, $\mathrm{SD}=1.55)$ than Armstrong $(\mathrm{M}=3.78, \mathrm{SD}=1.43)$, there were no significant differences [ $\mathrm{t}(164)=-1.78, \mathrm{p}=.63$ ], again suggesting that consumers could identify with both the self-inflicted hurts of Armstrong and those inflicted on Te'o.

While the first research question did not reveal any significant differences, support was found for the second hypothesis. An independent samples t-test was conducted to compare the means of sympathy scores for Armstrong and Te'o. There was a significant difference in sympathy scores for Armstrong $(\mathrm{M}=3.97, \mathrm{SD}=1.96)$ and Te'o $[\mathrm{M}=4.88, \mathrm{SD}=1.27 ; \mathrm{t} \quad(164)=-2.95, \mathrm{p}=.01 \quad$ (two-tailed) $]$. The magnitude in differences in the means (mean difference $=-.91,95 \% \mathrm{CI}$ : -1.53 to -.30$)$ indicated a moderate effect $(\eta 2=.05)$.

The third hypothesis proposed that participants would be more likely to view Te'o as naïve ( $\mathrm{H} 3 \mathrm{a})$ and gullible $(\mathrm{H} 3 \mathrm{~b})$ whereas Armstrong would be characterized as more manipulative $(\mathrm{H} 3 \mathrm{c})$ and cunning (H3d). Support was garnered for this multi-part hypothesis: Participants perceived Te'o $(\mathrm{M}=4.99, \mathrm{SD}=.26)$ as more naïve than Armstrong [(M=4.03, $\mathrm{SD}=.13), \mathrm{t}(164)=-2.67, \mathrm{p}=.04,2=.04]$, and also as more gullible [(Te'o; $\mathrm{M}=4.97, \mathrm{SD}=.23$; Armstrong; $\mathrm{M}=4.19, \mathrm{SD}=$. 15), $\mathrm{t}(164)=-2.63, \mathrm{p}=.01, \eta 2=.04]$. Similarly, findings indicate that Armstrong $(\mathrm{M}=3.81, \mathrm{SD}=1.29)$ is seen as more cunning [ $\mathrm{t}(164)=2.60$, $\mathrm{p}=.05, \eta 2=.04]$ than Te'o $(\mathrm{M}=2.78, \mathrm{SD}=1.10)$. Additionally, Armstrong $(\mathrm{M}=4.13, \mathrm{SD}=1.29)$ was seen as more manipulative than Te'o (M $=3.44, \mathrm{SD}=1.53),[\mathrm{t}(164)=2.66, \mathrm{p}=.01, \eta 2=.04]$.

The study's final research question sought to explore gender differences in regard to participants' identification with, empathy toward, and sympathy for, Armstrong and Te'o. Interestingly, no significant differences were found in levels of identification [men $(\mathrm{M}=3.93, \mathrm{SD}=1.16)$; women $(\mathrm{M}=3.61, \mathrm{SD}=1.25)]$, empathy [men $(\mathrm{M}=2.72, \mathrm{SD}=1.43)$; women $(\mathrm{M}=2.81, \mathrm{SD}=1.38)$ ], and sympathy [men $(\mathrm{M}=3.31, \mathrm{SD}=1.53)$; women $(\mathrm{M}=3.69, \mathrm{SD}=1.40)]$. This suggests that there are no statistically significant differences in the ways that men and women identify with or feel empathy and or sympathy to the two athletes in this study.

By and large, the authors found support for their hypotheses and research questions, indicating that news consumers do form emotional attachments to athletes, but these attachments appear to be subject to change based on the nature of the news attention surrounding the sports figure. The following discussion articulates how these findings impact theoretical and pragmatic implications.

\section{Discussion}

We compared the reactions to both Lance Armstrong and Manti Te'o of participants who self-identified as news consumers familiar with the respective scandals. In the main, the results corroborate recent research on ADT $[20,23]$ and the hero narrative, [4] while forwarding additional novel findings. Taken as a whole, the levels of identification, along with the earlier findings, confirm the validity of ADT, as participants did indicate significant levels of emotion toward the athletes. Our findings suggest that the hero narrative activates the human engine of emotion-even when participants do not identify particularly closely with an athlete. News consumers, at least those of the traditional college-going age, develop emotional attachments to athletes. One frequent and effective avenue for this development is the hero narrative, that familiar and predictable story arc that has trained news consumers to go beyond mere information seeking and also seek engagement with the main character.

This research is important theoretically because it reaffirms and extends ADT. Specifically, three of our six results significantly confirm ADT as it relates to parasocial interactions between audience and athlete. The participants in this study did report feeling emotions of empathy, sympathy and identification toward an athlete covered prominently for some time in the news; however, in regard to sympathy and empathy, it was not as significant as we expected. The egregiousness of Armstrong's behavior may have contributed to the relative lack of identification with him. Also, the relative youth and inexperience of Te'o may also have inclined consumers to a more positive affect toward him. Another element worth considering is that Armstrong, steeped as he was for so long in the hero narrative over a period of decades, had played out his hero narrative. Armstrong may have fulfilled the nine narrative sequences of the hero journey as described by Hoebeke, Deprez, and Raeymaeckers and [4] moved beyond the framework. To pursue this line of thinking, we note that Raney [14] explored the possibility that schemas may limit the breadth of affective dispositions a consumer might have for a character. Armstrong had lived under suspicion of drug use for years, and numerous news stories detailed the charges and Armstrong's responses. Perhaps the news coverage developed a story schema not of a hero, but of a cheater. Story schemas provide "moral-judgmental shortcuts that theoretically limit the application of the full affective continuum to characters" [14]. It could be that the strong positive feelings expressed by some participants for Te'o were in part due to the story schemas that limited other participants' affective disposition toward Armstrong. Taken together, both sets of responses confirm the development of parasocial relationships with athletes and illuminate the fact that news consumers not only bear witness to the victories and transgressions of athletes, but also affectively engage with sports figures.

In addition to offering theoretical benefits, this research also sparks some important pragmatic considerations. Pragmatically, this research 
is particularly important for practicing journalists and those in the public eye. For journalists, these findings indicate how memorable the hero narrative is to news consumers and the power of continued news coverage in developing parasocial interactions between consumers and news figures. As the data show, news consumers not only attend to the athletic prowess of a sports figure, but also familiarize themselves with the athlete's life as a whole - particularly when it involves a salacious scandal. To that end, it is essential that sports figures and celebrities are mindful of their actions on the field, but also in life because news consumers will attend to details to formulate judgments of the athlete. Such judgments have implications for the communication of athletes' personas in terms of sports marketing and public relations.

\section{Future Research and Limitations}

While this study did not include a content analysis of news coverage of Armstrong and Te'o, such analysis would further affirm the presence of the hero narrative. Also, the athletes examined were selected because the scandal involving each went public at approximately the same time; the authors acknowledge the athletes' drastic differences in terms of longevity, fan base and involvement in team versus individual sports.

Our findings contribute to the utility of the hero narrative paradigm in that they suggest that participants did tune into that narrative within the Armstrong and Te'o stories. The results also indicated participants' knowledge of the narrative arc of the athlete as hero via quantitative responses that affirmed affective disposition toward the characters of Te'o and Armstrong, though not always in significant amounts. For Te'o, his Internet stumble engendered considerable sympathy and some empathy, though not significantly more than for Armstrong. This data contributes to the already formidable body of ADT knowledge, adding information about a specific demographic -college-age consumers and the narrative messages they absorb.

A natural extension of the present study would include assessment of news consumers' perceptions of rule breaking by athletes who are positioned within the hero narrative by news coverage. Future analysis might investigate the effect of branding and the creation of a reservoir of goodwill by public relations professionals. Another avenue of further inquiry is to more closely examine the content of college students' social media use around this or other sports news coverage to ascertain how often re tweets and Facebook postings echo and retell the hero narrative put forth in the news media.

\section{References}

1. Lule J (2001) Daily news, eternal stories: The mythological role of journalism (Guilford Communication Series). Guilford Press.

2. Petersen B (2001) Celebrity, popular culture and sport. Youth Sport in Australia. Sydney: Sydney University Press.

3. Smart B (2005) The sport star: Modern sport and the cultural economy of the sporting celebrity. Thousand Oaks: Sage.

4. Hoebeke T, Deprez A, Raeymaeckers K (2011) Heroes in the sports pages: The troubled road to victory for Belgian cyclist Tom Boonen. Journalism Studies 12: 658-672.

5. Mandler JM (1984) Stories, Scripts, and Scenes: Aspects of Schema Theory. Hillsdale, NJ: Erlbaum.

6. Raney AA (2003) Disposition-based theories of enjoyment.

7. Zillmann D, Taylor K, Lewis K (1998) News as nonfiction theater: How dispositions toward the public cast of characters affect reactions. Journal of Broadcasting \& Electronic Media 42: 153-169.
8. Raney AA (2004) Expanding disposition theory: Reconsidering character liking, moral evaluations, and enjoyment. Communication Theory 14: 348-369.

9. Horne J (2005) Sport in consumer culture. Palgrave Macmillan.

10. Harper C, Parker K, Speight B, Dunne F (2013) Global sports media consumption report 2013. London: TV Sports Markets.

11. Lance Armstrong interview reaction: More skepticism than Sympathy (2013). Associated Press, CBS News.

12. Mandel S (2013) “Manti Te'o hoax should spark thirst for answers, not retribution.

13. O’Connell M (2013) TV ratings: 'Catfish' sees steady return, minus Manti Te'o buzz. The Hollywood Reporter.

14. Raney AA (2006) The psychology of disposition-based theories of media enjoyment. Psychology of entertainment. Lawrence Erlbaum Associates Publishers.

15. Knobloch-Westerwick S , Keplinger C (2007) Thrilling news: Factors generating suspense during news exposure. Media Psychology 9: 193-210.

16. Delgado F (2005) Golden but not brown: Oscar De La Hoya and the complications of culture, manhood, and boxing. The International Journal of the History of Sport 22: 196-211.

17. Potter WJ, Pashupati K, Pekurny RG, Hoffman E, Davis K (2002) Perceptions of television: A schema. Media Psychology 4: 27-50.

18. Ettema JS, Glasser TL (1988) Narrative form and moral force: The realization of innocence and guilt through investigative journalism. Journal of Communication 38: 8-26.

19. Berg LRV (1998) The sports hero meets mediated celebrity. NewYork: Routledge. 134-153.

20. Shafer DM, Raney AA (2012) Exploring How We Enjoy Antihero Narratives. Journal of Communication 62: 1028-1046.

21. Krakowiak KM, Oliver MB (2012) When good characters do bad things: Examining the effect of moral ambiguity on enjoyment. Journal of Communication 62: 117-135.

22. Sanders MS (2010) Making a good (bad) impression: examining the cognitive processes of disposition theory to form a synthesized model of media character impression formation. Communication Theory 20: 147-168.

23. Kinnally W, Tuzunkan F, Raney AA, Fitzgerald M, Smith J (2013) Using the Schema-Triggered Affect Model to Examine Disposition Formation in the Context of Sports News. Journal of Sports Media 8: 117-137.

24. Raney AA (2005) Punishing media criminals and moral judgment: The impact on enjoyment. Media Psychology 7: 145-163.

25. Raney AA (2011) The role of morality in emotional reactions to and enjoyment of media entertainment. Journal of Media Psychology 23: 18-23.

26. Baumeister RF, Leary MR (1995) The need to belong: Desire for interpersonal attachments as a fundamental human motivation. Psychological Bulletin 117: 497-529.

27. Fink JS, Parker HM, Brett M, Higgins J (2009) Off-field behavior of athletes and team identification: Using social identity theory and balance theory to explain fan reactions. Journal of Sport Management 23:142-155.

28. Kassing JW, Sanderson J (2009) "You're the Kind of Guy That We All Want for a Drinking Buddy": Expressions of Parasocial Interaction on Floydlandis. com.Western Journal of Communication 73: 182-203.

29. Horton D, Wohl RR (1956) Mass communication and para-social interaction: Observations on intimacy at a distance. Psychiatry. 19: 215-229.

30. Tian Q, Hoffner CA (2010) Parasocial interaction with liked, neutral, and disliked characters on a popular TV series. Mass Communication and Society 13: 250-269.

31. Brown WJ, Basil MD (2010) Parasocial interaction and identification: Social change processes for effective health interventions. Health Communication 25: 601-602.

32. Giles DC (2002) Parasocial interaction: A review of the literature and a model for future research. Media psychology 4: 279-305. 
Citation: Christian SE, Omilion-Hodges LM (2014) Emotional Reactions to Sports Heroes' Rise and Fall: Application of Affective Disposition Theory Via the Hero Narrative. J Mass Communicat Journalism 4: 201. doi:10.4172/2165-7912.1000201

Page 7 of 7

33. Cohen J (2001) Defining identification: A theoretical look at the identification of audiences with media characters. Mass Communication \& Society 4: 245-264.

34. Eyal K, Rubin AM (2003). Viewer aggression and homophily, identification, and parasocial relationships with television characters. Journal of Broadcasting \& Electronic Media 47: 77-98.

35. Stotland E, Mathews KE, Sherman SE, Hansson RO, Richardson BZ (1978) Empathy, fantasy, and helping. Beverly Hills: Sage Publications.

36. Cohen EL (2010) Expectancy violations in relationships with friends and media figures. Communication Research Reports 27: 97-111.
37. Gleeson SM (2013) Manti Te'o on girlfriend hoax story: 'victim' of 'sick joke', USA Today.

38. Manfred T (2013) NOTRE DAME: Manti Te'o was a victim of the dead girlfriend hoax. Business Insider.

39. McCroskey JC, McCain TA (1974) The measurement of interpersonal attraction. Speech Monographs, 41: 261-266.

40. Edwards C, Edwards A, Spence PR, Shelton AK (2014) Is that a bot running the social media feed? Testing the differences in perceptions of communication quality for a human agent and a bot agent on Twitter. Computers in Human Behavior 33: 371 - 376. 